\title{
Distributed detection of hydrogen and deuterium diffusion into a single-mode optical fiber with Chirped-Pulse $\varphi$ OTDR
}

\author{
A. Garcia-Ruiz,,$^{1,}$ A. Morana, ${ }^{2}$ L. $\operatorname{Costa}^{1}$ H.F. Martins, ${ }^{3}$ \\ S. MARTIn-Lopez, ${ }^{1}$ M. Gonzalez-Herraez, ${ }^{1}$ A. BOUKenter, ${ }^{2}$ \\ Y. OUERDANE, ${ }^{2}$ S. GIRARD ${ }^{2}$ \\ ${ }^{1}$ Departament of Electronics, University of Alcalá, Alcalá de Henares 28805, Spain \\ ${ }^{2}$ Univ Lyon, Laboratoire Hubert Curien, UMR CNRS 5516, 18 rue Professeur Benoit Lauras, 42000, Saint- Etienne, France \\ 3Instituto de Óptica "Daza de Valdés", IO-CSIC, C/Serrano 121, 28006 Madrid. \\ *Corresponding author: andres.garciaruiz@uah.es
}

Received XX Month XXXX; revised XX Month, XXXX; accepted XX Month XXXX; posted XX Month XXXX (Doc. ID XXXXX); published XX Month XXXX

For some infrastructures such as oil and gas extraction boreholes or radioactive waste repositories, where distributed optical fibre sensors are employed to grant the safety of the facilities, the presence of gas species such as hydrogen or deuterium is one of the most relevant parameters to monitor. The possibility of employing the same kind of sensors for this purpose is of special interest, reducing the cost by employing a single interrogator, able to measure multiple parameters by simply employing adequate sensing fibres. To meet this goal, we present here a chemical sensor based on Chirped-Pulse Phasesensitive Optical Time-Domain Reflectometry, which is able to detect these species while they diffuse into the silica fibre. The ability of Chirped-Pulse $\varphi$ OTDR to measure change of refractive index with a sensitivity around $10^{-8}$ has allowed determining hydrogen concentration with accuracy in the order of $10^{-3} \mathrm{~mol} / \mathrm{m}^{3}$ and spatial resolution $\sim 6 \mathrm{~m}$. Another experiment provided an indirect measurement of the solubility of deuterium in a standard telecom-grade optical fibre, that is found to be around $1.47 \times 10^{24} \mathrm{~m}^{3} / \mathrm{bar}$.

\section{(C) 2019 Optical Society of America}

http://dx.doi.org/10.1364/OL.99.099999

The use of optical fibres as a sensing element is currently a standard method when dealing with large infrastructures whose structural health must be permanently monitored due to the risks they involve[1]. The ability of distributed optical fibre sensors (DOFS) to measure at virtually every point along several kilometres-long structures is a major advantage of this class of sensors. DOFS present, in addition, all the good properties of optical fibre sensors, including a very good electromagnetic immunity, minimal intrusiveness of the sensor probe, and extremely good chemical, thermal and mechanical stabilities. These properties make DOFS an ideal solution to keep under control the temperature or vibrations of large critical facilities such as fossil fuel extraction rigs or nuclear waste repositories. In these industries, explosive gases can be released, and therefore optical fibres provide an additional advantage, as no electric power is required at the monitored points.

The idea of employing fibre sensors in order to detect and quantify the presence of gas species like hydrogen $\left(\mathrm{H}_{2}\right)$ is not new, and several approaches exist. They are usually based on transducers (such as palladium or platinum [2]) or other special coatings able to quickly react to the presence of the analyte and cause a mechanical strain in the sensing fibre, which can be easily detected by many DOFS technologies. However, this research topic continues open in order to achieve simpler (and cheaper) methods requiring only a standard telecom-grade optical fibre as a sensor [3], particularly in environments where the hydrogen concentration evolves slowly over time. This is the purpose of some research projects running at high level and intermediate level longlived radioactive waste repositories [4]. One of their current challenges concerns the identification of distributed gas sensors able to work in the harsh conditions associated with these facilities.

Thanks to the permeability of fused silica to small molecules such as $\mathrm{H}_{2}$ or $\mathrm{D}_{2}$ (deuterium), and to their known effect on the fundamental mode effective refractive index, their concentration along a conventional optical fibre could be directly measured using an adequate and sufficiently sensitive distributed sensor. Some of the DOFS in the state of the art present a coupled dependence on the gas concentration in the silica matrix. For example, those based on Brillouin scattering are not only affected by the change in refractive index, but also by the variation of the acoustic velocity of the 
medium [5]. However, in this letter we propose an alternative and effective method based on Rayleigh backscattering, whose working principles allow for direct measurement of the variations of the effective index of the guided mode. The method employed in this proof of concept, known as Chirped-Pulse Phase-sensitive Optical Time-Domain Reflectometry (CP- $\varphi$ OTDR), retrieves the local variation of the refractive index from shot to shot, interrogating at $\mathrm{kHz}$ repetition rates, and providing a linear transfer function between the measurand (typically strain or temperature) and the sensor outcome.

To understand how these molecules are introduced into the sensing fibre during the experiments realized, a brief introduction to the laws governing diffusion must be done. When the glass of the fibre is exposed to the considered analytes in a high-pressure atmosphere, the gas molecules start diffusing into the permeable fibre surface. This phenomenon can be described by a second order partial differential equation that determines the concentration of the gas molecules in space and time inside the host solid medium. In this equation, the gas is characterized by its diffusivity $(D)$, that is related to the absolute temperature (T), assumed homogeneous, through the Arrhenius equation [6]:

$$
D(T)=D_{\mathrm{o}} \exp \left(\frac{-E}{k T}\right) .
$$

In this expression, $E$ is the activation energy of the process and $k$ is Boltzmann's constant.

The solutions to the diffusion equation (1) for our cylindrical geometry, where the diffusion is forced to be radial starting from its external surface, is a sum of exponentials depending on time $(t)$ and modulated in space by Bessel functions [7]. In our experimental conditions, the progressive loading of the glass via radial diffusion can be phenomenologically described by a dominant term, leading to a simple exponential law for the evolution of the gas concentration (with non-constant term $\propto \pm \exp (-t / \tau)$ ), when considering only the fibre axis. As our sensing method is based on the propagation of light, mainly in the fibre core, we are mostly interested in this region. This qualitative behaviour holds for both the in-gassing and the out-gassing processes.

The characteristic time $(\tau)$ of the exponential law strongly depends on the fibre radius $(a)$, as it is proportional to $a^{2} / D$ [7]. Hence, it decreases with the experiment temperature $(T)$ via Eq. 1, but is not affected by the pressure $(P)$ of the surrounding atmosphere. The saturation value for the gas load, on the other hand, increases linearly with $P$ and decreases with $T$, as it also depends on the solubility $(S)$ of the gas in the silica glass. The solubility keeps the same functional relation with the temperature as the diffusivity (Eq. 1).

The presence of the diffused molecules in the silica matrix induces a noticeable effect on its dielectric susceptibility [7]. They are assumed to be incorporated interstitially, what is consistent with the linear relation between their concentration inside the fibre core and the variation of the refractive index of the glass, demonstrated by Swart et al.; for the saturated state, the expression of the refractive index perturbation $(\Delta n)$ is [7]:

$$
\Delta n=\frac{P\left(n_{\mathrm{X}}^{2}-1\right) S_{\mathrm{X}} R T}{2 P_{\mathrm{o}}^{2} N_{\mathrm{A}} n_{\mathrm{o}}},
$$

where $P$ is the pressure of the diffusing substance $(\mathrm{x}), n_{\mathrm{x}}$ is its gas-state refractive index, $S_{\mathrm{x}}$ is its solubility in fused silica at pressure $P_{\mathrm{o}}=1 \mathrm{~atm}, R$ is the gas constant, $N_{\mathrm{A}}$ is the Avogadro's number and $n_{\mathrm{o}}$ is the unperturbed refractive index of the fiber. This dependence of the fibre refractive index on the gas presence is the basis for the distributed sensor here proposed.

In addition to the effect of hydrogen on the fibre refractive index, its diffusion may also cause unwanted effects in the DOFS response. On one hand, diffusion of hydrogen in the fibre will cause absorption of light around $1240 \mathrm{~nm}\left(\mathrm{H}_{2}\right.$ overtone absorption band) and/or around $1380 \mathrm{~nm}$ after interaction with pre-existing point defects (due to the generated $\mathrm{OH}$ ), while also enhancing the mechanical fatigue of the glass. This will certainly have an impact in the range of the sensor. On the other hand, the change in refractive index recorded by hydrogen diffusion could also bias the outcome of the DOFS measurement, as it may be assumed as the result of a temperature or strain change. This would certainly happen for our technique (based on Rayleigh scattering) if no particular consideration is done. However, the mentioned detrimental effects of hydrogen diffusion can usually be prevented by the application of hermetic coatings (metals/carbon) to the sensing fibres [8], potentially allowing us to isolate the sole effect of the diffusing gas from other thermal or mechanical fluctuations in our setup, or in a realistic deployment of the proposed sensor.

In our experiment, three different single-mode fibre samples were spliced together, in separated loose coils around $30 \mathrm{~m}$ long. The gas-sensitive piece of fibre under test (FUT) was Corning ${ }^{\circledR}$ SMF-28 $\mathrm{e}^{\circledR}$ (with nominal refractive index $n_{\mathrm{o}}=1.47$, a diameter of $125 \mu \mathrm{m}$ and an acrylate primary coating). After it, two types of hermetic fibres were spliced: a segment of pure-silica fibre with a carbon coating (Fibertronix ${ }^{\circledR}$ ) and a Hole-Assisted Carbon-Coated (HACC) fibre sample, described in [9]. These two fibres only measured index variations due to the experiment temperature evolution, providing a reference curve to be subtracted from the gas signal in order to distinguish the effect of diffusion in the permeable sample, as suggested above. At the output end of the fibre, a few centimetres-long coreless fibre was spliced, in order to avoid unwanted reflections back to the interrogator.

The prepared FUT was placed inside a sealed high-pressure, stainless steel vessel (Fig. 1) provided with feedthroughs for fibres of different diameters or other instrumentation, as well as a manometer. The reactor was then filled with hydrogen at a pressure around 150 bars. This provides a wide dynamic range for the refractive index modulation, as gas pressure increases the saturation load of the glass [5]. For the desorption, the hydrogen partial pressure in the vessel was nulled by replacing its content with nitrogen (unable to diffuse into the silica glass), avoiding mechanical effects on the measurement due to a change in the external pressure. A similar procedure was followed with the deuterium, which was kept at 100 bars. The temperature of the whole experiment was around $25^{\circ} \mathrm{C}$ (non-controlled, room temperature).

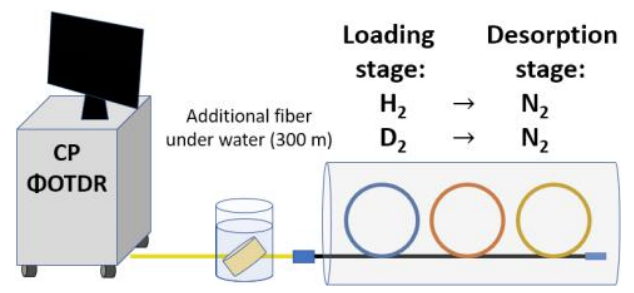

Fig.1 Diagram of the high-pressure gas reactor and FUT: two hermetic and a permeable SMF samples simultaneously exposed to the analytes. 
The CP- $\varphi$ OTDR setup employed for the interrogation of the FUT is similar to a conventional $\varphi$ OTDR system, but the pulses emitted present a linear evolution on their instantaneous frequency, what translates the measurand variations not into local trace amplitude variations, but into local trace time shifts [10]. These principles allow the achievement of high sensitivity and single-shot, linear readout, allowing to track dynamic $(\mathrm{kHz})$ changes of refractive index with sensitivities around $10^{-8}$ and spatial resolutions of a few meters.

The relation between the local shifts $\left(\left.\Delta t\right|_{z}\right)$ found when comparing consecutive traces in the regions $(z)$ where a perturbation has been applied is given by [10]:

$$
\left.\Delta t\right|_{z}=\frac{v_{\mathrm{o}}}{\delta v} \tau_{\mathrm{p}} \frac{\Delta n(z)}{n},
$$

where $v_{\mathrm{o}}$ and $\delta v$ are the spectral center and span of the chirped pulses, respectively, and $\tau_{\mathrm{p}}$ their duration. $\left.\Delta t\right|_{z}$ can be measured by simply cross-correlating corresponding regions from consecutive traces. For this reason, the method is perfectly suited to distributed acoustic sensing. However, by tracking the cumulative $\left.\Delta t\right|_{z}$ it is also possible to carry out longer term measurements, as it is proved with the experiment presented here.

The time shifts, as derived from the CP- $\varphi$ OTDR theory, are originated exclusively by the refractive index variation or strain applied to the fibre, being sensitive to temperature changes. However, for the hermetic samples, $\Delta n$ will only be related to a change in the fibre local temperature. To subtract these variations from the data registered at the permeable segment, we applied an experimental coefficient that relates a temperature gradient with the $\Delta n$ it induces, whose value is $-6.9210^{-6} \mathrm{~K}^{-1}$ [11].

The employed optical setup is shown in Fig. 2. In the upper branch, the chirped pulses are generated. This is done by an external cavity laser diode (LD) source. It generates a continuous wave (CW) of wavelength $1550 \mathrm{~nm}$, controlled by stabilizing its driving current and temperature (I,T). To chirp the signal, a sawtooth modulation is applied to the LD bias current. A synchronous gating square waveform is sent to a semiconductor optical amplifier (SOA), resulting in $6 \mathrm{~m}$ long ( $60 \mathrm{~ns}$ ) pulses with a chirp bandwidth of $\sim 600 \mathrm{MHz}$, that will be launched to the FUT at a repetition rate of $4 \mathrm{kHz}$. The pulses are amplified at an erbiumdoped fibre amplifier (EDFA) whose amplified stimulated emission (ASE) is filtered out prior to the emission of the pulses.

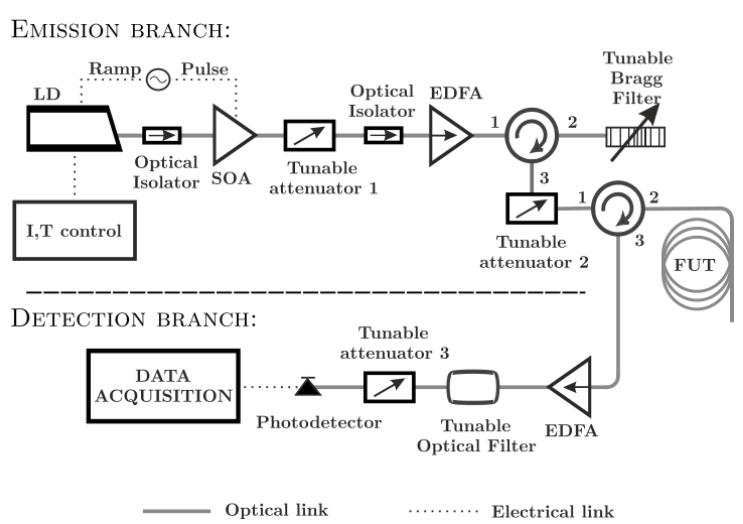

Fig.2 Optical setup inside the employed experimental interrogator. Acronyms are explained within the text.
The detection branch is provided with an amplifying and filtering stage similar to the one described before, in order to condition the signal for the fast $\mathrm{p}$-i-n photodetector used. The detector bandwidth matches the chirp bandwidth. The produced electrical signal is finally digitized at a rate of 2 GSps and processed by a computer. This parameter and the pulse length and chirp applied determine the sensitivity of the system to refractive index changes, which is in the order of $10^{-8}$, according to Eq. 3. In this experiment (lasting several days) a technique to mitigate the effect of the laser phase noise was implemented [12]. To do this, an additional $300 \mathrm{~m}$ long fibre segment was placed at the FUT input end (see Fig. 1). By keeping it at a stable temperature (under water), the developed interrogator can subtract laser frequency fluctuations from the actual refractive index changes undergone in the fibres [12].

In this experiment, 5 measurement points were monitored in each fibre sample. On the other hand, the possibility of measuring with similar gauge length in ranges up to $75 \mathrm{~km}$ have been previously demonstrated for this technique [13]. The data registered during the hydrogen experiment at representative points of each fibre sample is shown in Fig. 3 (top). The curves measured at points in the hermetic samples correspond to actual room temperature drifts. The $\sim 5^{\circ} \mathrm{C}$ periodic cycles must be compared to the gas-induced index variation, equivalent to an apparent temperature modulation of more than $100^{\circ} \mathrm{C}$. The smoother relative measurement plotted corresponds to the refractive index change in the permeable fibre with the day and
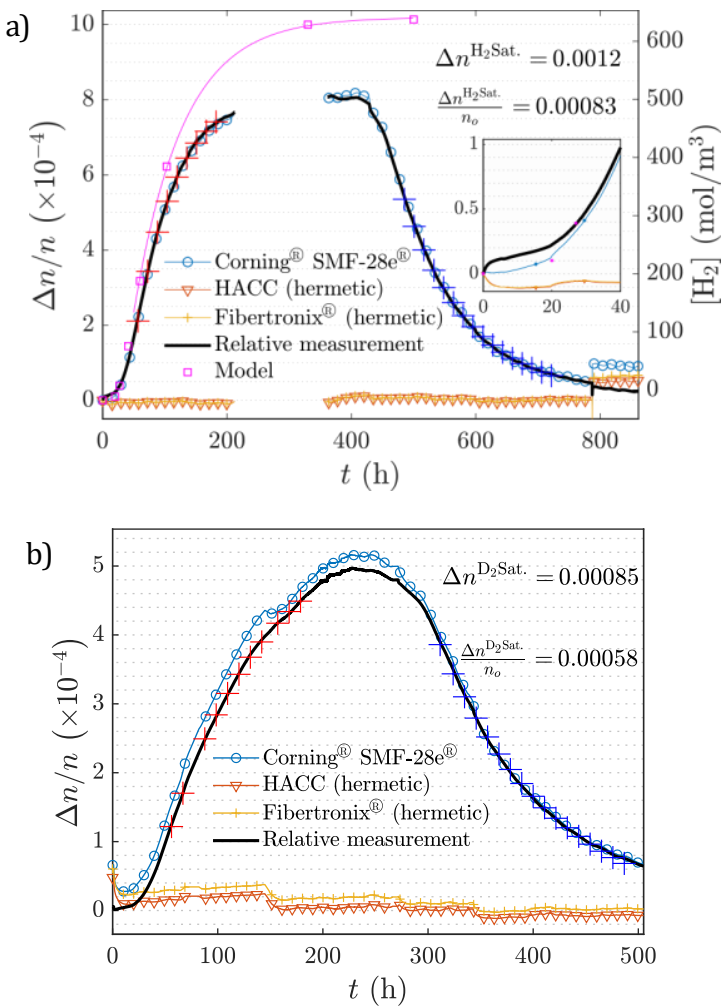

Fig. 3. a) measured fiber effective relative $\Delta n$ while being loaded with $\mathrm{H}_{2}$ at $\sim 150$ bars. A theoretical calculation is shown for comparison. Red and blue crosses represent exponential fits. b) the equivalent experiment for the same fiber samples now exposed to $D_{2}$ at $\sim 100$ bars. 
night temperature oscillations subtracted. As can be seen in the inset, the main effect observed during the first $\sim 24 \mathrm{~h}$ is due to the sudden initial increase in the hydrostatic pressure applied to the fibre. During this first day, the huge gas concentration gradient driving the process forced the first gas molecules to travel radially across the cladding, to finally reach the core, where their effect on refractive index becomes detectable. Although the exposure time (interrupted merely by a temporal fibre break outside the vessel, which did not affect the gas diffusion process) was not enough to reach the saturation of the load and the relaxation process was not tracked until desorption was completed, the nominal refractive index is almost recovered at the end of the experiments, demonstrating the long-term stability of the method.

The models describing the saturation concentration [7] and the corresponding induced refractive index change (Eq. 2), as well as the coefficients found in reference [14] for $\mathrm{H}_{2}$, provide the pink curve in Fig. 3 (top) and allow us to map the measured index change to a hydrogen concentration, $\left[\mathrm{H}_{2}\right]$, as shown in the right axis. These experimental results also provide an estimation of the sensitivity of the method to hydrogen concentration in the fused silica fibre core. Given the refractive index sensitivity of the interrogator system, the minimum measurable concentration is around $10^{-3} \mathrm{~mol} / \mathrm{m}^{3}$.

To characterize the observed loading and desorption processes, the relative measurement curve was fitted to exponentials (red and blue crosses). The obtained coefficients provide a saturation value for the refractive index variation of $\Delta n^{\mathrm{H}_{2} \mathrm{Sat}}=1.2 \times 10^{-3}$, that corresponds to a hydrogen concentration in the bulk silica glass around $500 \mathrm{~mol} / \mathrm{m}^{3}$. The resulting diffusion characteristic time (averaged from absorption and desorption) is $\tau\left(\mathrm{H}_{2}\right)=3.37 \pm 0.2$ days.

The discrepancy with the presented model is especially noticeable in the saturation value $(+20 \%)$. This could be related to the variability of the coefficients for the theoretical calculations. Parameters such as the solubility of the gas in the fibre have demonstrated a high dependence on the specific composition of the glass employed for its fabrication, as well as on the thermal history of the particular studied specimen [14]. However, the characteristic time, which depends on the gas diffusivity (Eq.1) and the fibre radius, is in good agreement with the model ( $-2 \%)$.

In Fig. 3 (bottom), the results corresponding to the deuterium experiment are plotted. Due to the reduced literature on diffusion of deuterium in fused silica glass [6], the measurement is not compared with the model in this case. On the other hand, we can take advantage of the proposed sensor to estimate the solubility of deuterium in the Corning ${ }^{\circledR}$ fibre glass, by applying Eq. 2. Considering the exponential fitting, the required saturation refractive index change is $\Delta n^{\mathrm{D}_{2} \text { Sat. }}=8.5 \times 10^{-4}$. By taking $n_{\mathrm{D}_{2}} \simeq n_{\mathrm{H}_{2}}$, we obtain a value for the solubility of $1.47 \cdot 10^{24} \mathrm{~m}^{-3} / \mathrm{bar}$. This number corresponds to a $57 \%$ of the value employed for the hydrogen diffusion model.

As for the characteristic diffusion time of deuterium, the fitting coefficients provide $\tau\left(\mathrm{D}_{2}\right)=4.28 \pm 0.4$ days. This result is consistent with a lower diffusivity of $\mathrm{D}_{2}$ in the fibre glass, as expected from the smaller mass of hydrogen molecules.

In conclusion, the proposed distributed sensor has proven the possibility of detecting and quantifying the presence of $\mathrm{H}_{2}$ and $\mathrm{D}_{2}$ along a conventional optical fiber. Considering the results, the sensor based on CP- $\varphi$ OTDR could represent a competitive method when compared to those in the state of the art, especially in environments where concentrations are only slowly evolving, the diffusion time of $\mathrm{H}_{2}$ into the fibre being then not a limiting factor.
The combination of a hermetic and a permeable fibre allows separating and determining independently the gas concentration and temperature fluctuations suffered by the fibre. The achieved sensitivity to hydrogen is estimated around $10^{-3} \mathrm{~mol} / \mathrm{m}^{3}$, according to the concentration axis. Comparable data of deuterium properties is not available. Conversely, we provide an estimated value of $1.47 \cdot 10^{24} \mathrm{~m}^{-3} / \mathrm{bar}$ for its solubility in fused silica, as obtained from the measurements. This result fits the experimental curve to the theoretical diffusion kinetics behaviour.

For applications interested in pressures below our experimental regime, the saturation level will be linearly decreased. However, even for levels close to atmospheric pressure, the reported sensitivity should be enough to detect and quantify the tested species after a few hours of exposure if appropriate temperaturereferencing fibres are implemented and packed close to the permeable fibre. This setup also grants simultaneous monitoring of the temperature of the target facilities. As an example, the approximately linear increase found during the first $10 \mathrm{~h}$ of exposure to hydrogen shown in Fig. 3 (top) corresponds to a rate of $0.4 \mathrm{~mol} /\left(\mathrm{m}^{3} \mathrm{~h}\right)$. To enhance the response time of the sensor, the use of fibres with outer diameters smaller than $125 \mu \mathrm{m}$ could be advisable, as well as other specialty fibres where silica-path for the molecules could be smaller (e.g. certain PCFs). This would reduce the time molecules take to reach the fibre core from the fibre external surface and increase the ratio between surface exposed to the gas pressure and volume of silica glass to load.

Acknowledgments. The authors thank Dr. S. Delepine-Lesoille for her collaboration with the models and interpretation of the results.

Funding. European Commission (EC) (H2020) and Ministerio de Economía y Competitividad (MINECO) (DOMINO, ERANET cofounded Water Works 2014 call); EC (FINESSE project, MSCAITN-ETN-722509); MINECO (TEC2015-71127-C2-2-R); Ministerio de Ciencia, Innovación y Universidades (MICINN) (IJCI-201733856, RTI2018-097957-B-C31); Comunidad de Madrid (CM) and FEDER program (SINFOTON2-CM: P2018/NMT-4326); University of Alcalá (FPI grant).

\section{References}

1. J.M. López-Higuera, L.R. Cobo, et al., J. Lightw. Technol. 29, 587 (2011).

2. M. Aleixandre, P. Corredera, et al., Sensors Actuat. B, 107, 113 (2005).

3. S. Delepine-Lesoille, J. Bertrand, et al., IEEE Photon. Technol. Lett. 24, 1475 (2012).

4. S. Delepine-Lesoille, S. Girard, et al., Sensors, 17, 1377 (2017).

5. S. Leparmentier, J.L. Auguste, et al., in 24th International Conference on Optical Fibre Sensors (International Society for Optics and Photonics, 2015), vol. 9634, p. 963432.

6. J. Stone, J. of Lightw. Technol. 5, 712 (1987).

7. P.L. Swart, A.A. Chtcherbakov, et al., Opt. Commun. 217, 189 (2003).

8. P. J. Lemaire and E. A. Lindholm, in Specialty optical fibers handbook (Academic Press, 2007), ch. 14.

9. S. Girard, D. Di Francesca, et al., IEEE Transactions on Nuclear Science, 62, 2941 (2015).

10. J. Pastor-Graells, H.F. Martins, et al., Opt. Express 24, 13121 (2016).

11. Y. Koyamada, M. Imahama, et al., J. Lightw. Technol. 27, 1142 (2009).

12. M.R. Fernández-Ruiz, J. Pastor-Graells, et al., J. Lightw. Technol. 36, 979 (2018).

13. J. Pastor-Graells, J. Nuño, et al., J. Lightw. Technol. 35, 4677 (2017).

14. J.F. Shackelford, P.L. Studt, and R.M. Fulrath, J. Appl. Phys. 43, 1619 (1972). 


\section{Full references (APA):}

1. López-Higuera, J. M., Cobo, L. R., Incera, A. Q., \& Cobo, A. (2011). Fiber optic sensors in structural health monitoring. Journal of Lightwave Technology, 29(4), 587-608.

2. Aleixandre, M., Corredera, P., Hernanz, M. L., \& Gutierrez-Monreal, J. (2005). Development of fiber optic hydrogen sensors for testing nuclear waste repositories. Sensors and Actuators B: Chemical, 107(1), 113-120.

3. Delepine-Lesoille, S., Bertrand, J., Lablonde, L., \& Pheron, X. (2012). Distributed hydrogen sensing with Brillouin scattering in optical fibers. IEEE Photonics Technology Letters, 24(17), 1475-1477.

4. Delepine-Lesoille, S., Girard, S., Landolt, M., Bertrand, J., Planes, I., Boukenter, A., Marin, E., Humbert, G., Leparmentier, S., Auguste, J.L., \& Ouerdane, Y. (2017). France's state of the art distributed optical fibre sensors qualified for the monitoring of the French underground repository for high level and intermediate level long lived radioactive wastes. Sensors, 17(6), 1377.

5. Leparmentier, S., Auguste, J. L., Humbert, G., Pilorget, G., Lablonde, L., \& Delepine-Lesoille, S. (2015, September). Study of the hydrogen influence on the acoustic velocity of single-mode fibers by Rayleigh and Brillouin backscattering measurements. In 24th International Conference on Optical Fibre Sensors (Vol. 9634, p. 963432). International Society for Optics and Photonics.

6. Stone, J. (1987). Interactions of hydrogen and deuterium with silica optical fibers: A review. Journal of Lightwave technology, 5(5), 712-733.

7. Swart, P. L., Chtcherbakov, A. A., Joubert, W. L., \& Shlyagin, M. G. (2003). Study of the pressure dependence of hydrogen diffusion in optical fiber by an interferometric technique. Optics communications, 217(1-6), 189-196.

8. Lemaire, P. J., \& Lindholm, E. A. (2007). Hermetic optical fibers: carboncoated fibers. Specialty optical fibers handbook, 453-490.

9. Girard, S., Di Francesca, D., Boukenter, A., Robin, T., Marin, E., Ladaci, A., ... \& Planes, I. (2015). On-site regeneration technique for hole-assisted optical fibers used in nuclear facilities. IEEE Transactions on Nuclear Science, 62(6), 2941-2947.

10. Pastor-Graells, J., Martins, H. F., Garcia-Ruiz, A., Martin-Lopez, S., \& Gonzalez-Herraez, M. (2016). Single-shot distributed temperature and strain tracking using direct detection phase-sensitive OTDR with chirped pulses. Optics express, 24(12), 13121-13133.

11. Koyamada, Y., Imahama, M., Kubota, K. and Hogari, K. (2009). Fiberoptic distributed strain and temperature sensing with very high measurand resolution over long range using coherent OTDR. J. Lightw. Technol., 27(9), 1142-1146.

12. Fernández-Ruiz, M. R., Pastor-Graells, J., Martins, H. F., Garcia-Ruiz, A., Martin-Lopez, S., and Gonzalez-Herraez, M. (2018). Laser phase-noise cancellation in chirped-pulse distributed acoustic sensors. J. Lightw. Technol., 36(4), 979-985.

13. Pastor-Graells, J., Nuño, J., Fernández-Ruiz, M. R., Garcia-Ruiz, A., Martins, H. F., Martin-Lopez, S., \& Gonzalez-Herraez, M. (2017). Chirped-pulse phase-sensitive reflectometer assisted by first-order Raman amplification. Journal of Lightwave Technology, 35(21), 46774683.

14. Shackelford, J. F., Studt, P. L., \& Fulrath, R. M. (1972). Solubility of gases in glass. II. $\mathrm{He}, \mathrm{Ne}$, and $\mathrm{H} 2$ in fused silica. Journal of Applied Physics, 43(4), 1619-1626. 